\title{
Tuberculosis in Patients with Primary Myelofibrosis During Ruxolitinib Therapy: Case Series and Literature Review
}

This article was published in the following Dove Press journal: Infection and Drug Resistance

\author{
Yizhou Peng (D) \\ Li Meng' \\ Xuemei $\mathrm{Hu}^{2}$ \\ Zhiqiang $\mathrm{Han}^{3}$ \\ Zhenya Hong'
}

'Department of Hematology, Tongji Hospital, Tongji Medical College, Huazhong University of Science and Technology, Wuhan, Hubei, People's Republic of China; ${ }^{2}$ Department of Radiology, Tongji Hospital, Tongji Medical College, Huazhong University of Science and Technology, Wuhan, Hubei, People's People's Republic of China; ${ }^{3}$ Cancer Biology Research Center, Tongji Hospital, Tongji Medical College, Huazhong University of Science and Technology, Wuhan, Hubei, People's Republic of China
Correspondence: Zhenya Hong Department of Hematology, Tongji Hospital, Tongji Medical College, Huazhong University of Science and Technology, 1095 Jie-Fang Avenue, Wuhan 430030, Hubei, People's Republic of China $\mathrm{Tel} / \mathrm{Fax}+86$ 027-83665046

Email hongzhenya@I26.com

Zhiqiang Han

Cancer Biology Research Center, Tongji

Hospital, Tongji Medical College,

Huazhong University of Science and

Technology, 1095 Jie-Fang Avenue, Wuhan 430030, Hubei, People's Republic of China Email hanzq2003@126.com
Background: The selective Janus-activated kinase inhibitor ruxolitinib (rux) is now widely used to treat myelofibrosis and polycythemia vera due to its remarkable effect of reducing splenomegaly and improving constitutional symptoms. With opportunistic infections secondary to rux constantly reported; however, an increasing number of studies have begun to investigate the mechanism and underlying immunosuppressive effect of rux.

Case Presentation: We report two cases of tuberculosis (TB) in primary myelofibrosis patients during rux therapy. The first patient received rux soon after diagnosis, and tracheobronchial TB (TBTB) and bronchoesophageal fistula were found after 4 months. After discontinuation of rux, antituberculosis therapy (ATT) was introduced. The second patient initiated rux due to progressive splenomegaly after 7.5 years of interferon therapy and was diagnosed with disseminated TB after 2 months. He received ATT as well. His rux was maintained due to the high burden of systematic symptoms and splenomegaly. Both myelofibrosis and TB were well controlled in these patients.

Conclusion: This is the first case report that describes rux-related TBTB accompanied by a bronchoesophageal fistula. Through a review of the literature, we provide supporting evidence to the finding that intrinsic disorders of myeloproliferative neoplasms and rux-induced immunologic deregulation together lead to TB. We highlight the importance of screening for latent TB infection and timely chemoprophylaxis before rux therapy. Once TB is diagnosed during treatment, rux is recommended to be stopped and active ATT should begin quickly.

Keywords: myelofibrosis, ruxolitinib, tuberculosis, infection

\section{Introduction}

Primary myelofibrosis (PMF), a stem cell-derived myeloproliferative neoplasm (MPN), is often characterized by the presence of bone marrow fibrosis, anemia, splenomegaly and constitutional symptoms, such as fatigue, night sweats, weight loss, and pruritus. The Janus kinase-signal transducer and activator of transcription (JAK-STAT) pathway is known to play a crucial role in cell proliferation and differentiation. As the most frequent genetic mutation in MPN, the JAK2V617F mutation is present in approximately $96 \%$ of patients with polycythemia vera (PV), $55 \%$ with essential thrombocythemia (ET), and 65\% with PMF. ${ }^{1}$ Targeting this pathway, ruxolitinib (rux), a selective JAK1 and 2 inhibitor, is applied to reduce splenomegaly and improve the series of constitutional symptoms, and has achieved significant effects. However, opportunistic infections associated with rux have been reported since its launch, suggesting a latent immunosuppressive effect. 
Here, we report two cases of tuberculosis (TB) discovered during rux treatment for PMF patients and intend to provide practicable prophylaxis and treatment by reviewing the literature and analyzing our clinical experience.

\section{Case Presentation}

\section{Case I}

A 41-year-old male came to the hospital for fatigue, satiety and abdominal distension in March 2018. Palpation revealed that the lower edge of his spleen reached the umbilicus level. His chest computed tomography (CT) scan showed no abnormality. Bone marrow biopsy showed remarkably increased age-adjusted cellularity with granulocyte proliferation, decreased erythropoiesis, a large number of megakaryocytes atypia, grade 0 fibrosis, and the JAK2V617F mutation. He was diagnosed with Pre-PMF. His Dynamic International Prognostic Scoring System (DIPSS) risk group was intermediate-1. He was started on rux (20 mg, twice daily).

Fever and productive cough started in early July and lasted approximately one week. He was hospitalized on 12th July for antibiotics. Laboratory tests showed white blood cells (WBC) $16.11 \times 10^{9} / \mathrm{L}$, erythrocyte sedimentation rate (ESR) $40 \mathrm{~mm} / \mathrm{h}$, hypersensitive C-reactive protein $107.7 \mathrm{mg} / \mathrm{L}$, and procalcitonin (PCT) $0.76 \mu \mathrm{g} / \mathrm{L}$. Blood lymphocyte subsets showed an absolute total $\mathrm{T}$ cell count of 347/ $\mu \mathrm{L}$ (normal range: $995-2860 / \mu \mathrm{L}$ ) and $\mathrm{CD}^{+} \mathrm{T}$ cell count of $208 / \mu \mathrm{L}$ (normal range: $550-1440 / \mu \mathrm{L}$ ). Sputum acid-fast bacilli (AFB) smears were negative, but interferon gamma release assay (IGRA, T-SPOT.TB) was positive. Transient syncope unexpectedly occurred the next morning. Multiple deep venous thrombosis was later confirmed by ultrasound in his left lower extremity. Enhanced CT showed pulmonary thromboembolism in his right upper and inferior pulmonary artery, bilateral pleural effusion, pulmonary infection, multiple enlarged mediastinal lymph nodes and soft tissue shadows that surrounded his left main bronchus (Figure 1A and C). CT also revealed frontal lobe hemorrhage. He received low molecular weight heparin instantly, and rux was discontinued from then on. To prevent the occurrence of lethal complications, he was transferred to vascular surgery for inferior vena cava filter implantation. A bronchoesophageal fistula on his left main bronchus was later discovered by iodine contrast esophagogram in response to persistent dry cough, and then he received nasal jejunal nutrition (Figure 1B).
Hydroxyurea was introduced to control the WBC count in September. Given that infection work-up was negative, fiberoptic bronchoscopy was chosen to determine whether he was infected with TB. However, bronchoalveolar lavage fluid (BALF) specimens tested negative for culture, AFB and GeneXpert assay. A tracheobronchial lymph node biopsy finally confirmed Mycobacterium tuberculosis (MTB) by polymerase chain reaction. He was diagnosed with tracheobronchial tuberculosis (TBTB) and received a 12-month antituberculosis therapy (ATT) involving isoniazid, rifampicin, ethambutol and levofloxacin. Another two bronchoalveolar lavages were performed during ATT. BALF was negative for all relevant results, including mycobacterial ribonucleic acid (RNA) and deoxyribonucleic acid (DNA). The mediastinal lymph nodes' size also decreased (Figure 2). In July 2019, rux (10 mg, twice daily) was reintroduced due to splenomegaly and abdominal distension. His DIPSS was intermediate-1 at that time.

\section{Case 2}

A 66-year-old male was diagnosed with PMF in December 2010 at a local hospital. Bone marrow biopsy showed megakaryocytic proliferation, grade 3 fibrosis and the JAK2V617F mutation. Because rux was not available in China at that time, he received regular interferon therapy for 7.5 years. To mitigate the progression of splenomegaly, rux (20 mg, twice daily) monotherapy started in late June 2018. The DIPSS risk group was intermediate-2. The single dose was adjusted to $25 \mathrm{mg}$ in early August owing to an unsatisfactory reduction in spleen size.

Unexplained fever started on 20th August and lasted until September (Tmax $\left.39.2^{\circ} \mathrm{C}\right)$. Laboratory tests showed WBC $14.14 \times 10^{9} / \mathrm{L}$, hemoglobin $78 \mathrm{~g} / \mathrm{L}$, platelet $198 \times 10^{9} /$ L, PCT $0.65 \mu \mathrm{g} / \mathrm{L}$, and ESR $60 \mathrm{~mm} / \mathrm{h}$. Blood samples were positive for T-SPOT.TB and TB antibody. His purified protein derivative (PPD) test was strongly positive. Chest CT scan presented small diffuse nodules in the bilateral lungs, pleural effusion, adhesion, and enlarged lymph nodes in the retroperitoneum. He was diagnosed with disseminated TB (DTB).

His temperature gradually stabilized when 12 months of ATT (isoniazid, levofloxacin, rifapentine and amikacin) started in October 2018. Considering the potential progression of PMF, rux (20 mg, twice daily) was retained under close monitoring of liver function. Nevertheless, accompanied by the reappearance of fever, his left hip joint began to hurt, and a neck mass was found by palpation on the same side. Imageology examinations confirmed the existence of 

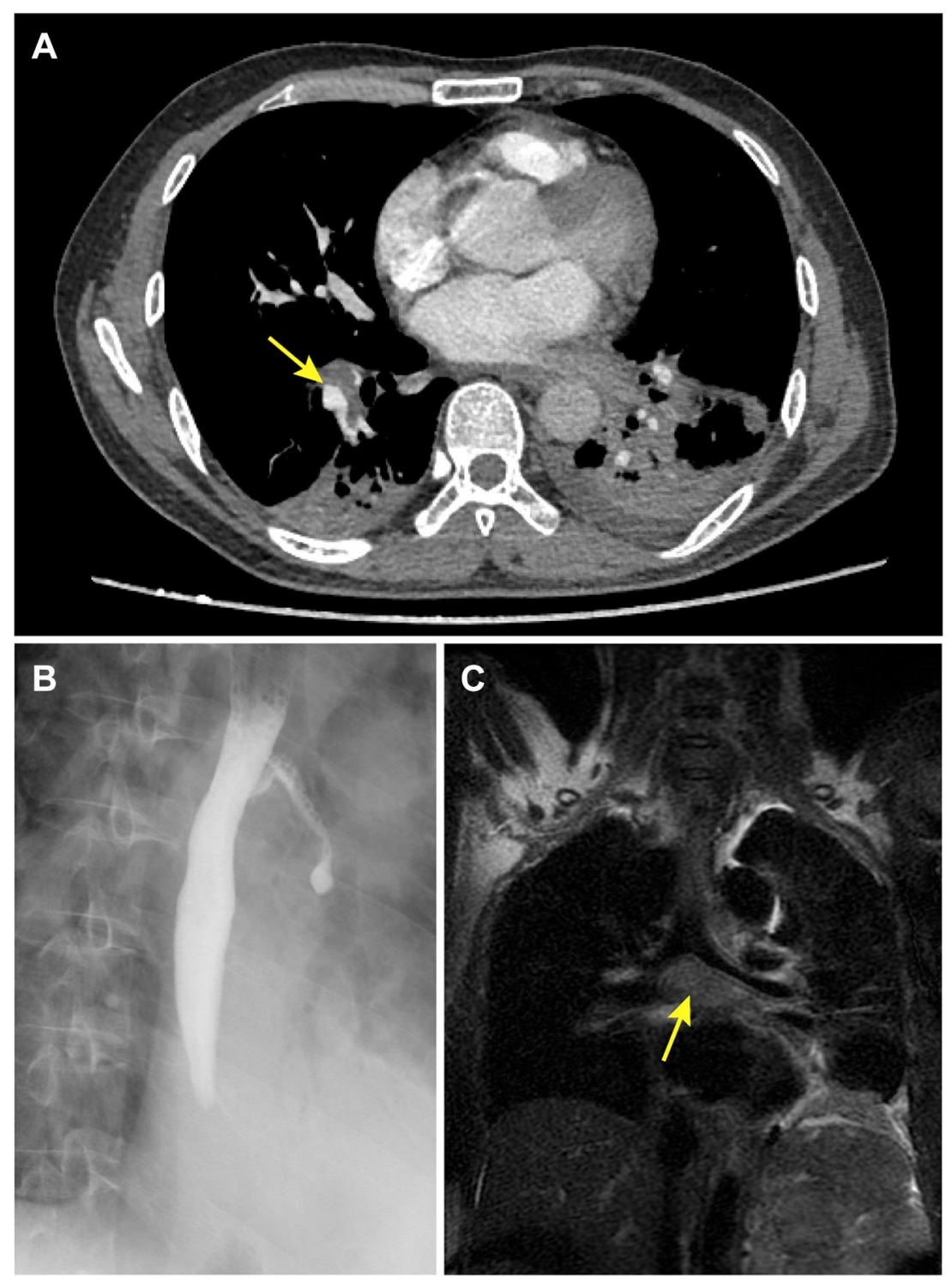

Figure I Imaging appearances in case I. (A) Enhanced chest computed tomography (CT) showing the pulmonary thromboembolism (arrow) in right inferior pulmonary artery and bilateral pleural effusion. (B) lodine contrast esophagogram confirmed the bronchoesophageal fistula. (C) Coronal magnetic resonance imaging (MRI) showed the mediastinal soft tissue shadows (arrow) surrounded the left main bronchus.

neck and bilateral psoas abscesses and Schmorl's nodes at L4 and L5 (Figure 3). During debridement, a $3 \times 15.6 \times 2.8 \mathrm{~cm}$ focus on the left, a $3 \times 10 \times 2 \mathrm{~cm}$ focus on the right, and a $4 \times 3 \mathrm{~cm}$ focus of the neck were found. All three contained yellow pus and caseous necrosis, in which the DNA and RNA of MTB were detected as positive. Amikacin was replaced by ethambutol in November.

\section{Discussion}

The selective JAK1/2 inhibitor rux is widely used for its potent effect on reducing splenomegaly and improving systemic symptoms. Focusing on its efficiency and safety, a series of clinical trials were performed. In the 5-year follow-up analysis of the phase $ш$ COMFORT- I trial, a significantly increased infectious rate was not found in the rux group when compared with the control group (except herpes zoster infection). ${ }^{2}$ Similar outcomes were detected in other trials. ${ }^{3,4}$ However, sporadic opportunistic infection cases related to rux have been reported worldwide since 2012.

Drug-induced impacts aside, studies have noted the abnormal baseline immune status of MPN. Published works demonstrate that the count of regulatory $\mathrm{T}$ cells has decreased before the introduction of rux, which may contribute to a pro-inflammatory status and disrupt the immune surveillance of myelofibrosis (MF) patients. $^{5,6}$ 

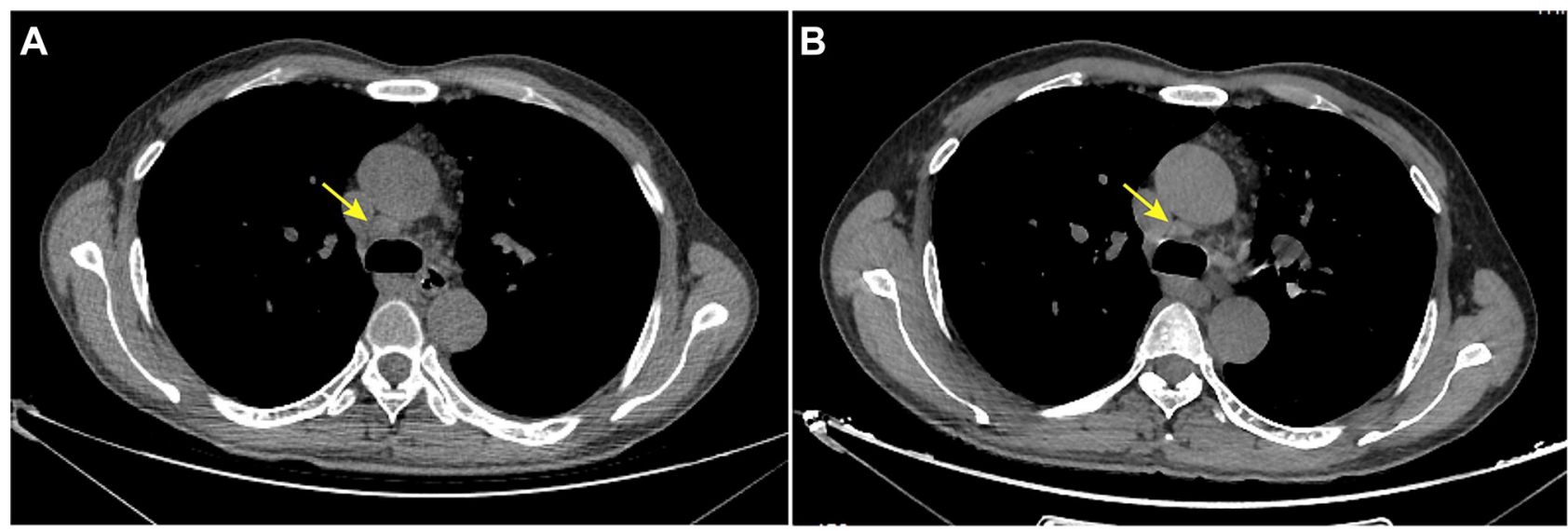

Figure 2 CT images of case I in September (A) and November (B). The enlarged mediastinal lymph node (arrow) decreased after antituberculosis therapy.
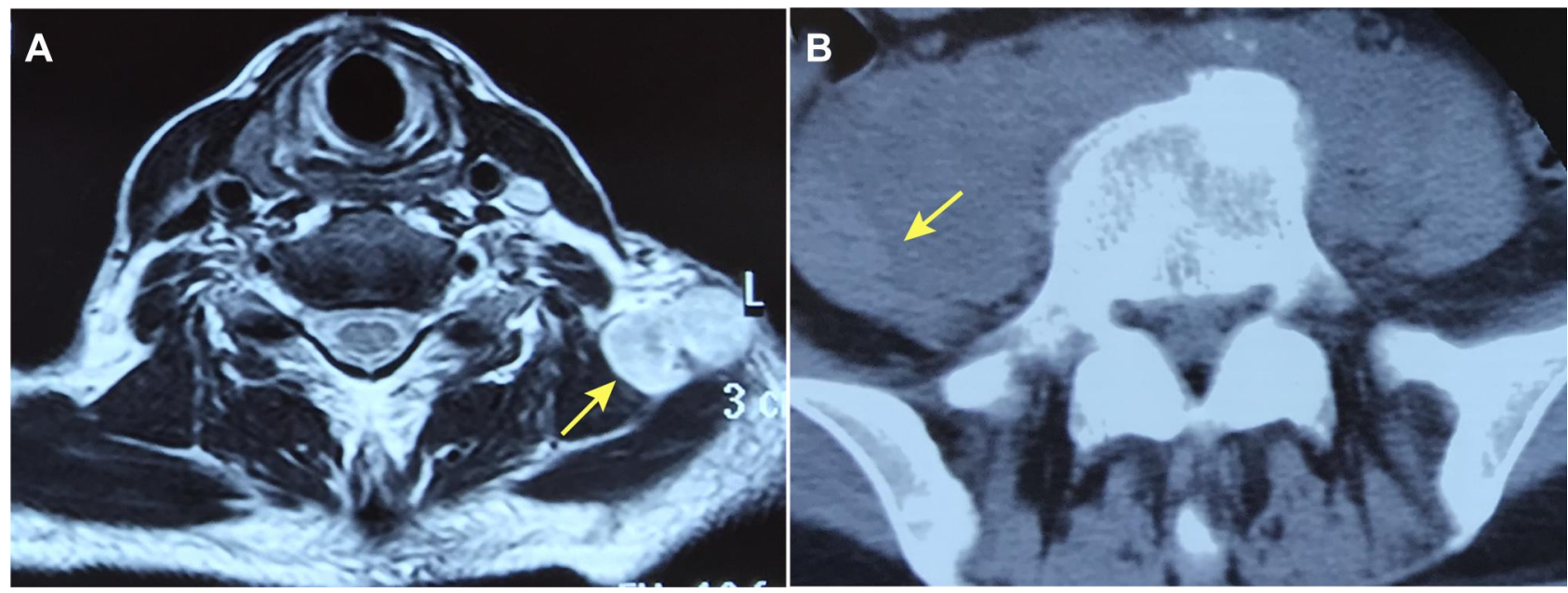

Figure 3 Imaging appearances in case 2. (A) The neck abscess (arrow) on axial MRI. (B) The right psoas abscess (arrow) on axial CT.

Although rux is seen as a selective inhibitor, its inhibitory effect is not confined to JAK1 and 2 as it can influence dozens of other kinases to varying degrees. ${ }^{7}$ The consequent "off-target" effect causes different immune disorders. Data suggest that rux potently inhibits the differentiation and function of dendritic cells. ${ }^{8}$ Rux can also impair the killing ability of natural killer cells. ${ }^{9}$ In cell-mediated immunity, the drug negatively affects $\mathrm{T}$ helper 1 (Th1) cell function and downregulates interferon gamma (IFN- $\gamma$ ) and tumour necrosis factor- $\alpha$ (TNF$\alpha$ ), the cytokines that play a crucial role in the resistance against MTB. ${ }^{10,11}$ In line with the findings, the absolute values of the $T$ lymphocyte subsets were significantly decreased in the first patient. The alteration induced by rux can offset some treatment benefits and trigger TB. A recent study that focused on the MF patients revealed that the risk of TB was two times higher in the rux-treated group. ${ }^{12}$ Likewise, in patients treated with anti-TNF- $\alpha$ agents for autoimmune diseases, the risk of developing TB increases approximately fourfold. ${ }^{13}$ It should also be noted that common targeted therapies in hematological malignancy, such as rituximab (anti-CD20 monoclonal antibody), do not lead to a significant increase in TB. ${ }^{14}$

The change in infectious rate is not always obvious. ${ }^{15,16}$ We note that the incidence of herpes zoster infection is significantly increased in the rux group, while other infections are not. ${ }^{2}$ Revealing the same outcome, a systematic review suggested a clinical relevance to the risk. ${ }^{17}$ Published data showed a predisposition to infections in MF patients. ${ }^{18}$ Additionally, in a recent study, patients who received rux were all at intermediate-2/high IPSS risk, a factor that independently correlates with a higher infectious risk, since older age and advanced disease status contribute to a poorer clinical condition. ${ }^{19}$ 
It is difficult to draw the conclusion that rux alone causes more infectious events when those identified confounding factors and other potential ones exist. Therefore, we believe that intrinsic disorders of MPN and rux-induced immunologic deregulation together lead to the occurrence of atypical and opportunistic infections involving bacteria, viruses and fungi. Whether and to what degree these confounding factors affect infectious risk needs further exploration.

We intend to analyze the few existing cases to understand the basic clinical characteristics of TB during rux treatment. By the end of April 2020, 23 cases of ruxrelated TB were described in 17 published case reports (Table 1). ${ }^{20-36}$ Our two cases are listed in this table and will be analyzed as well.

Males constituted the majority of patients $(60 \%)$, and the average age of the whole group was $65.8 \pm 11.5$ years old. All patients were diagnosed with MF aside from one with $\mathrm{PV}$. As the most common diagnosis in this group, $20(80.0 \%)$ patients had extrapulmonary $\mathrm{TB}$, while simple pulmonary TB accounted for the remaining $20.0 \%$. The duration of rux before infection varied from 3 weeks to 47 months and was mainly concentrated in the first 6 months, with a median time of 5 months. Fever was the most common initial symptom but was not specific. All patients were subsequently treated with ATT except two cases for which data were unavailable. Although rux was discontinued for the majority of patients after the TB diagnosis, there were also four cases (including case 2) that directly used ATT and achieved improved clinical symptoms. ${ }^{21,28}$ In seven patients (including case 1), rux was successfully reintroduced due to the relapse of systematic symptoms and/or splenomegaly. Long-term isoniazid monotherapy was maintained in one patient for prophylaxis after standard ATT, despite the lack of signs of TB reactivation. ${ }^{25}$ Three patients had a history of prednisolone before or during rux therapy, and one of them had also used lenalidomide for about 17 months. ${ }^{23,31,33}$ Several patients had concomitant diabetes, chronic liver disease or tumours, all of which could lead to different degrees of immune disorder and infection susceptibility. Screening for latent TB infection (LTBI) was only performed for five patients (including case 1). Neither further examination nor treatment for LTBI was planned for the only patient whose IGRA was positive. ${ }^{33}$ Eight patients (32.0\%), including the only atypical mycobacterial infection (M. avium complex), finally died. ${ }^{34}$ The young PV patient was the only one who experienced allogeneic transplantation after resolving TB and was clinically stable in the post-transplant period. ${ }^{31}$
It is difficult to determine whether the TB was primary or reactivation because most cases had not been screened before and had an unknown TB history. Nevertheless, elderly patients have more chances of being exposed to TB. It is possible that their immune systems were once strong enough to respond to MTB and led TB into a latent stage. Under the dual influence of MPN and rux, the balanced, relative static state between host and MTB is broken, which results in a rapid reproduction of bacilli in granulomas. ${ }^{37}$ Active MTB can be released into a patient's airway, lymphatic system or bloodstream and then travel to a distant place, thus causing more extrapulmonary $\mathrm{TB}$ in a short time. China is listed as one of the 30 countries with a high TB burden by the World Health Organization and accounts for $9 \%$ of the global total. Although our patients denied past infection and nothing abnormal was found on $\mathrm{CT}$ in case 1, we are still inclined to support TB reactivation when taking the high TB burden in China and a relatively short onset time after rux therapy into account. The incidence of TB might even be higher than studies have reported, since not all infectious events can be identified. In patients who have been previously treated with other immunomodulators, laboratory examinations can give false-negative results, thus missing the chance to recognize them. Moreover, a longer rux exposure may be needed for atypical mycobacterial infection. ${ }^{34}$

Infection is one of the major causes of morbidity and mortality in MF patients. Therefore, it is of great importance to distinguish patients at higher risk of developing TB and implement prophylactic measures and treatment. A comprehensive assessment of infectious risk at first can help to individualize prevention methods. It is strongly recommended that every patient be screened with IGRA (T-SPOT.TB or QuantiFERON-TB) and chest X-ray for LTBI before the initiation of rux. The tuberculin skin test alone is not advised by us here for its low specificity but can serve as a supplementary test. If diagnosed, patients need to delay rux therapy and start a single-drug ATT with isoniazid promptly, which works well in several cases. ${ }^{38}$ Similar measures have decreased the risk of developing TB in patients who received anti-TNF- $\alpha$ agents. ${ }^{39}$ Regular re-examination and follow-up are required during rux usage. Co-prescription with steroids or other immunomodulatory agents is not suggested owing to the underlying dual immunosuppressive effect. ${ }^{40}$ Since symptoms of TB are unspecific and similar to MF, clinicians should be vigilant to ensure that no possible symptoms are missed and to make differential diagnoses when they appear so 


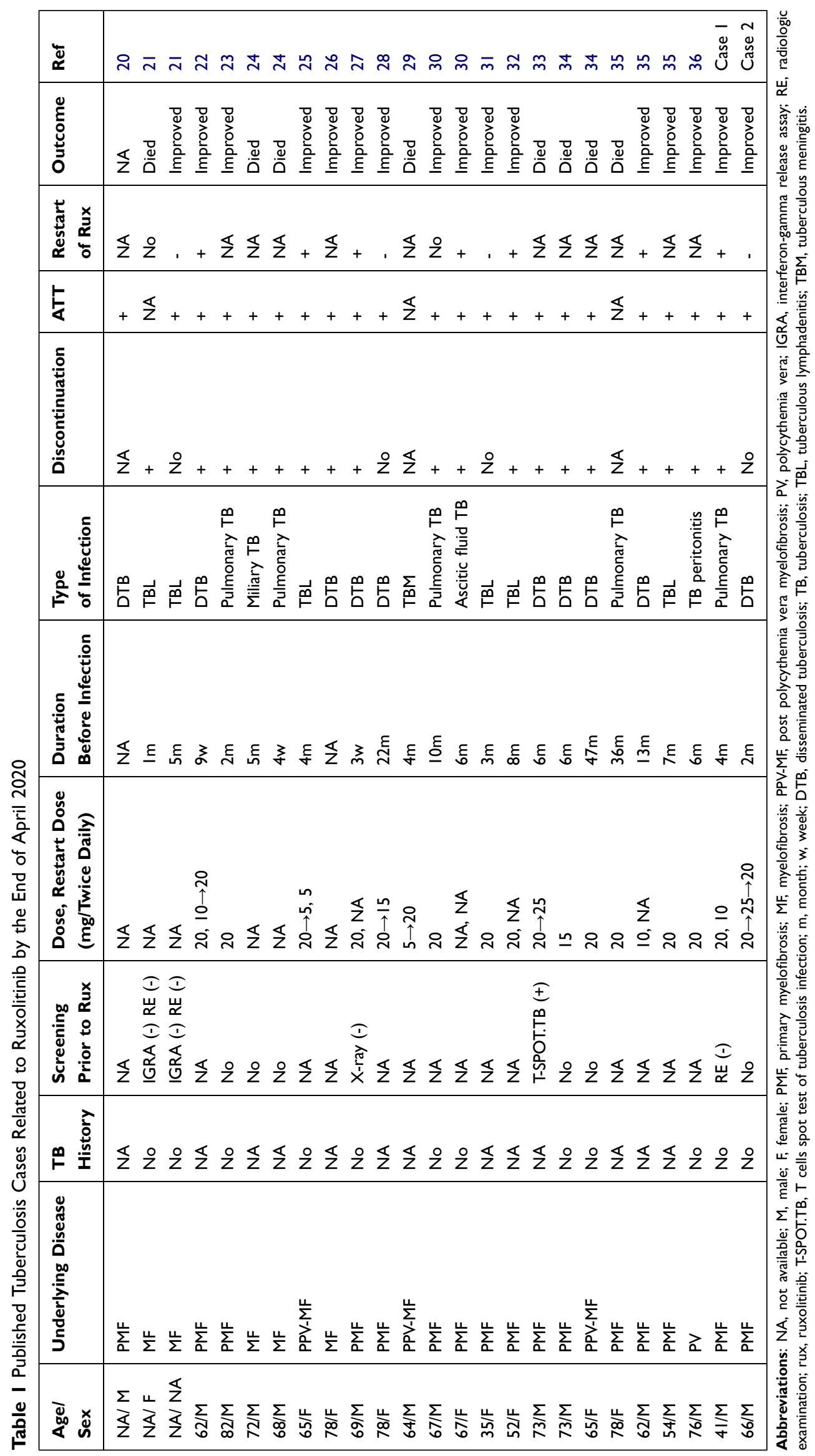


that treatment can start at an early stage. It is reasonable to discontinue rux because its immunosuppressive effect may aggravate infection. All studies indicate that an active ATT should be started as soon as possible. Reintroduction of rux mostly depends on the relapse of constitutional symptoms and splenomegaly because they may denote a possibility of progression.

\section{Conclusion}

We reported the first TBTB complicated by a bronchoesophageal fistula in a rux-treated PMF patient. LTBI screening and prophylactic ATT (if necessarily) are recommended for candidates for rux. Since there are few relevant cases, the recommendations are mainly concluded from the clinical experience of finite resources and require further investigation to test their efficiency. What needs to be emphasized is that opportunistic infections should not become the reason to limit the application of rux, given the substantial benefit the drug has brought to patients in the past few years.

\section{Ethical Statement}

Written informed consents were obtained from both patients for publication of the case details and images. This study was approved by the Medical Ethics Committee of the Department of Hematology, Tongji Hospital, Tongji Medical College, Huazhong University of Science and Technology (TJ-IRB20190705).

\section{Funding}

This work was supported by the National Natural Science Foundation of China [Grant Nos. 81873430, 81974414, and 81772788].

\section{Disclosure}

The authors have no conflicts of interest to declare for this work.

\section{References}

1. Tefferi A. Primary myelofibrosis: 2019 update on diagnosis, risk-stratification and management. Am J Hematol. 2018;93(12):15 51-1560. doi:10.1002/ajh.25230

2. Verstovsek S, Mesa RA, Gotlib J, et al. Long-term treatment with ruxolitinib for patients with myelofibrosis: 5-year update from the randomized, double-blind, placebo-controlled, Phase 3 COMFORT-I trial. J Hematol Oncol. 2017;10(1):55. doi:10.1186/s13045-017-0417-z

3. Harrison CN, Vannucchi AM, Kiladjian JJ, et al. Long-term findings from COMFORT-II, a phase 3 study of ruxolitinib vs best available therapy for myelofibrosis. Leukemia. 2016;30(8):1701-1707. doi:10. 1038/leu.2016.148
4. Vannucchi AM, Kiladjian JJ, Griesshammer M, et al. Ruxolitinib versus standard therapy for the treatment of polycythemia vera. $N$ Engl J Med. 2015;372(5):426-435.

5. Romano M, Sollazzo D, Trabanelli S, et al. Mutations in JAK2 and calreticulin genes are associated with specific alterations of the immune system in myelofibrosis. Oncoimmunology. 2017;6(10): e1345402. doi:10.1080/2162402X.2017.1345402

6. Wang JC, Sindhu H, Chen C, et al. Immune derangements in patients with myelofibrosis: the role of Treg, Th17, and sIL2Ralpha. PLoS One. 2015;10(3): $\mathrm{e} 0116723$.

7. Rudolph J, Heine A, Quast T, et al. The JAK inhibitor ruxolitinib impairs dendritic cell migration via off-target inhibition of ROCK. Leukemia. 2016;30(10):2119-2123. doi:10.1038/leu.2016.155

8. Heine A, Held SA, Daecke SN, et al. The JAK-inhibitor ruxolitinib impairs dendritic cell function in vitro and in vivo. Blood. 2013;122 (7):1192-1202.

9. Schonberg K, Rudolph J, Vonnahme M, et al. JAK inhibition impairs NK cell function in myeloproliferative neoplasms. Cancer Res. 2015;75(11):2187-2199. doi:10.1158/0008-5472.CAN-14-3198

10. Parampalli Yajnanarayana S, Stübig T, Cornez I, et al. JAK1/2 inhibition impairs $\mathrm{T}$ cell function in vitro and in patients with myeloproliferative neoplasms. $B r J$ Haematol. 2015;169(6):824-833. doi:10.1111/bjh.13373

11. Keohane C, Kordasti S, Seidl T, et al. JAK inhibition induces silencing of $\mathrm{T}$ Helper cytokine secretion and a profound reduction in T regulatory cells. Br J Haematol. 2015;171(1):60-73. doi:10.1111/ bjh. 13519

12. Polverelli N, Breccia M, Benevolo G, et al. Risk factors for infections in myelofibrosis: role of disease status and treatment. A multicenter study of 507 patients. Am J Hematol. 2017;92(1):37-41. doi:10.1002/ ajh. 24572

13. Baddley JW, Cantini F, Goletti D, et al. ESCMID Study Group for Infections in Compromised Hosts (ESGICH) Consensus Document on the safety of targeted and biological therapies: an infectious diseases perspective (Soluble immune effector molecules [I]: anti-tumor necrosis factor-alpha agents). Clin Microbiol Infect. 2018;24(Suppl 2):S10-S20. doi:10.1016/j.cmi.2017.12.025

14. Mikulska M, Lanini S, Gudiol C, et al. ESCMID Study Group for Infections in Compromised Hosts (ESGICH) Consensus Document on the safety of targeted and biological therapies: an infectious diseases perspective (Agents targeting lymphoid cells surface antigens [I]: CD19, CD20 and CD52). Clin Microbiol Infect. 2018;24 (Suppl 2):S71-S82. doi:10.1016/j.cmi.2018.02.003

15. Tremblay D, King A, Li L, et al. Risk factors for infections and secondary malignancies in patients with a myeloproliferative neoplasm treated with ruxolitinib: a dual-center, propensity score-matched analysis. Leuk Lymphoma. 2019;61(3):660-667.

16. Komatsu N, Kirito K, Shimoda K, et al. Assessing the safety and efficacy of ruxolitinib in a multicenter, open-label study in Japanese patients with myelofibrosis. Int J Hematol. 2017;105(3):309-317. doi:10.1007/s12185-016-2130-z

17. Lussana F, Cattaneo M, Rambaldi A, Squizzato A. Ruxolitinibassociated infections: a systematic review and meta-analysis. $\mathrm{Am}$ J Hematol. 2018;93(3):339-347. doi:10.1002/ajh.24976

18. Hultcrantz M, Wilkes SR, Kristinsson SY, et al. Risk and cause of death in patients diagnosed with myeloproliferative neoplasms in Sweden between 1973 and 2005: a population-based study. J Clin Oncol. 2015;33(20):2288-2295. doi:10.1200/JCO.2014.57.6652

19. Polverelli N, Palumbo GA, Binotto G, et al. Epidemiology, outcome, and risk factors for infectious complications in myelofibrosis patients receiving ruxolitinib: a multicenter study on 446 patients. Hematol Oncol. 2018;36(3):561-569. doi:10.1002/hon. 2509

20. Colomba C, Rubino R, Siracusa L, et al. Disseminated tuberculosis in a patient treated with a JAK2 selective inhibitor: a case report. $B M C$ Res Notes. 2012;5:552. doi:10.1186/1756-0500-5-552 
21. Kim Y-K, Lee SR, Park Y, et al. Efficacy of ruxolitinib in Korean myelofibrosis patients and cases complicated TB lymphadenitis during the treatment. Blood. 2013;122(21):1596. doi:10.1182/blood. V122.21.1596.1596

22. Hopman RK, Lawrence SJ, Oh ST. Disseminated tuberculosis associated with ruxolitinib. Leukemia. 2014;28(8):1750-1751. doi:10.10 38/leu.2014.104

23. Chen $\mathrm{YH}$, Lee $\mathrm{CH}$, Pei SN. Pulmonary tuberculosis reactivation following ruxolitinib treatment in a patient with primary myelofibrosis. Leuk Lymphoma. 2015;56(5):1528-1529.

24. Keizer S, Gerritsen R, Jauw Y, Janssen J, Koopman B, Bresser P. Fatal tuberculosis during treatment with ruxolitinib. Ned Tijdschr Geneeskd. 2015;159:A8650-A8650.

25. Palandri F, Polverelli N, Catani L, Vianelli N. Ruxolitinib-associated tuberculosis: a case of successful ruxolitinib rechallenge. Ann Hematol. 2015;94(3):519-520. doi:10.1007/s00277-014-2183-0

26. Shamil E, Cunningham D, Wong BLK, Jani P. Ruxolitinib associated tuberculosis presenting as a neck lump. Case Rep Infect Dis. 2015;2015:1-3. doi:10.1155/2015/284168

27. Abidi MZ, Haque J, Varma $P$, et al. Reactivation of pulmonary tuberculosis following treatment of myelofibrosis with ruxolitinib. Case Rep Hematol. 2016;2016:2389038.

28. Branco B, Metsu D, Dutertre M, et al. Use of rifampin for treatment of disseminated tuberculosis in a patient with primary myelofibrosis on ruxolitinib. Ann Hematol. 2016;95(7):1207-1209. doi:10.1007/ s00277-016-2684-0

29. Malkan UY, Haznedaroglu IC. A myelofibrosis case that develops mycobacterial infection after ruxolitinib treatment. Int J Clin Exp Med. 2017;10(4):7304-7307.

30. Neethu C, James J, Prabhu R. Reactivation of a common infection following treatment with a novel agent for an uncommon disease-ruxolitinib associated tuberculosis: two cases. J Pharm Sci Res. 2017;9(12):2437-2439.

31. Patil V, Wasekar N, Jijina F, Mohite A. Ruxolitinib-associated tuberculosis - a rare complication of a novel drug! Int J Med Sci Public Health. 2017;6(3):1. doi:10.5455/ijmsph.2017.0846912092016
32. Pepeler MS, Ozkurt ZN, Guzel OT, Akyurek N. Tuberculosis reactivation related with ruxolitinib in a patient with primary myelofibrosis. J Infect Dev Ctries. 2018;12(10):926-928. doi:10.38 55/jidc.9993

33. Tsukamoto Y, Kiyasu J, Tsuda M, et al. Fatal disseminated tuberculosis during treatment with ruxolitinib plus prednisolone in a patient with primary myelofibrosis: a case report and review of the literature. Intern Med. 2018;57(9):1297-1300. doi:10.2169/internalmedicine.91 65-17

34. Lescuyer S, Ledoux MP, Gravier S, et al. Tuberculosis and atypical mycobacterial infections in ruxolitinib-treated patients with primary or secondary myelofibrosis or polycythemia vera. Int $J$ Infect Dis. 2019;80:134-136. doi:10.1016/j.ijid.2019.01.002

35. Khalid F, Damlaj M, AlZahrani M, Abuelgasim KA, Gmati GE. Reactivation of tuberculosis following ruxolitinib therapy for primary myelofibrosis: case series and literature review. Hematol Oncol Stem Cell Ther. 2020. doi:10.1016/j.hemonc.2020.02.003

36. Sakiyama E, Chinen Y, Tsukamoto T, et al. Tuberculosis peritonitis during treatment of polycythemia vera with ruxolitinib. Infect Drug Resist. 2020;13:1017-1021. doi:10.2147/IDR.S249030

37. Russell DG, Cardona PJ, Kim MJ, Allain S, Altare F. Foamy macrophages and the progression of the human tuberculosis granuloma. Nat Immunol. 2009;10(9):943-948. doi:10.1038/ni.1781

38. Heine A, Brossart P, Wolf D. Ruxolitinib is a potent immunosuppressive compound: is it time for anti-infective prophylaxis? Blood. 2013;122(23):3843-3844. doi:10.1182/blood-2013-10-531103

39. Favalli EG, Desiati F, Atzeni F, et al. Serious infections during anti-TNFalpha treatment in rheumatoid arthritis patients. Autoimmun Rev. 2009;8(3):266-273. doi:10.1016/j.autrev.2008.11. 002

40. Warzocha K, Homenda W, Pluta A, et al. Ruksolitynib w terapii chorych na mielofibrozę - pytania i odpowiedzi. Hematologia. 2019;9(4):269-284. doi:10.5603/Hem.2018.0035
Infection and Drug Resistance

\section{Publish your work in this journal}

Infection and Drug Resistance is an international, peer-reviewed openaccess journal that focuses on the optimal treatment of infection (bacterial, fungal and viral) and the development and institution of preventive strategies to minimize the development and spread of resistance. The journal is specifically concerned with the epidemiology of antibiotic resistance and the mechanisms of resistance development and diffusion in both hospitals and the community. The manuscript management system is completely online and includes a very quick and fair peerreview system, which is all easy to use. Visit http://www.dovepress.com/ testimonials.php to read real quotes from published authors. 